\title{
Cortical generators of normal electroencephalographic waveforms: A literature review
}

\author{
Pooja Ojha ${ }^{1, *}$, Nitin Pandey ${ }^{2}$, Seema Singh ${ }^{3}$ \\ ${ }^{1}$ Assistant Professor, ${ }^{2}$ Associate Professor, ${ }^{3}$ Professor, Dept. of Physiology, Era's Lucknow Medical College, Lucknow, Uttar \\ Pradesh, India
}

*Corresponding Author:

Email: drpojha@gmail.com

Received: $15^{\text {th }}$ December, 2017

Accepted: $5^{\text {th }}$ April, 2018

\begin{abstract}
Introduction: EEG is a useful, noninvasive, safe technique to study the electrical activity of brain in various physiological and pathological conditions like alertness, sleep, seizure activity. Normal brain rhythms, the electroencephalogram (EEG) represents the electrical activity of brain as recorded through the metal electrodes applied over scalp. An insight to the possible source of EEG, various rhythms and its activation during certain maneuvers can give a better understanding to its applications.

Materials and Methods: Number of electronic database, as well as library resources were searched for related references included in published literature till 2017, using following key words 'cortical', 'electroenchephalogram',' EEG', EEG Rhythm', EEG wave', 'alpha wave',' beta wave',' theta wave',' delta wave'. Those which were related to our subject were included in the review.

Results: Of the total number of studies we have finally included 35 studies from search results. These are mentioned in the references.

Conclusion: Beta, Alpha, Theta \& Delta are produced from various regions of the brain, including cortical \& thalamic networks, hippocampus, reticular networks and so on. The rhythm of EEG can be correlated with the level of activation of the brain, with beta wave being produced during alert state and delta wave being produced during deep sleep. Interventions like hyperventilation and photic stimulation can be used to increase the diagnostic yield of EEG. It has been seen that sex-related differences do exist in the degree of lateralization of hemispheric function.
\end{abstract}

Keywords: Electroencephalogram, Brain waves, Cortex, Thalamus, EEG.

\section{Introduction}

The electroencephalogram (EEG) is the record of electrical activity of brain obtained through the metal electrodes applied over scalp with the help of conductive paste. ${ }^{1}$ It is the difference in voltage between two different recording locations plotted over time. Electroencephalographic recording is a noninvasive procedure which can be repeated on the patients without any risk. Introduction of EEG to the pool of investigations for brain has been a boon to mankind. It has helped researchers to know a lot about the functioning of brain in health and disease. An insight to the possible source of EEG, various rhythms and its activation during certain manoeuvres can give a better understanding to its possible applications. This work intends to provide a literature review from past and recent times which will throw light upon the foresaid queries.

\section{Materials and Methods}

Number of electronic database, as well as library resources were searched for related references included in published literature till 2017, using following key words 'cortical', 'electroenchephalogram', EEG', EEG Rhythm',' EEG wave', 'alpha wave',' beta wave',' theta wave',' delta wave'. Manual search was also done to find related references from books and journals. Those studies which were duplicated or had irrelevant content or failed to provide any detail were excluded. 35 studies, which had relevant content to our subject were finally included in the review.

\section{Discussion}

This section is described in four subheadings. First part of article presents the details of origin of EEG, second section has details of classification of brain waves. Followed by EEG during sleep and various interventions in third subheading and lastly variation in EEG with gender.

Origin of EEG: It was Caton who, more than a hundred years ago found that there is electrical activity occurring in the brain. ${ }^{2}$ In the year 1920 , Berger demonstrated that it is possible to record this electrical activity from the surface of the brain with the help of Electroencephalogram (EEG). Only after this EEG became popular as a modality to study the normal and abnormal functioning of brain. $^{3}$ The Electroencephalography reached a new stage when in the year 1934, two renowned scientists Adrian and Matthews confirmed the concept of human brain waves in a paper and they were also successful in observing oscillations with a frequency of $10-12 \mathrm{~Hz}$. It was later termed as "alpha rhythm". ${ }^{4}$

Classification of Brain Waves: Electroencephalographic rhythms are defined as regularly recurring waveforms of similar shape and duration. These rhythms have been identified since times when EEG was recorded in humans and animals. It is observed that there is intrinsic firing property in 
many thalamic, thalamocortical and cortical neurons, which enables them to form a cellular network generating rhythmic EEG activity. The basic rhythms of brain can be identified if the subject remains relaxed with eyes closed. These rhythms appear as sinusoidal patterns, ranging in amplitude from $0.5-100 \mu \mathrm{v}$ when measured from peak to peak. The broad categories of brain rhythms are Beta / gamma oscillations $(>13 \mathrm{~Hz})$, Alpha $(8-13 \mathrm{~Hz})$, Theta $(4-8 \mathrm{~Hz})$, Delta $(0.5-4 \mathrm{~Hz})$.

Beta Oscillations: Hans Berger, a German Psychiatrist found the Beta rhythm of the brain in 1924. In the somatomotor cortex, beta oscillations were described in the awake cat by the research group of Buser and Rougeul-Buser (2005) particularly when the animal was in a state of enhanced vigilance while watching an unreachable mouse. ${ }^{5}$ Also, fast oscillations were found in the somato motor cortex in monkey during a state of enhanced attention. ${ }^{6}$ Oscillations of $25-35 \mathrm{~Hz}$ occurred in the sensorimotor cortex of awake, behaving monkeys in local field potentials and single-/multiunit recordings. They were particularly apparent during the performance of motor tasks that required fine finger movements and focussed attention. The physiological beta frequencies may be broken down as follows: a) Frontal beta: which is fairly common and may be very fast, without any relationship to physiological rhythm. b) Central beta: this is often mixed with other rhythms. c) Posterior beta: this is often a fast alpha equivalent, reactive like alpha rhythm. d) Diffuse beta: not linked to any special physiological rhythm.

Alpha Rhythm: In an animal that is relaxed but awake, Alpha rhythm can be recorded from posterior aspect of brain typically from the occipital area. Alpha waves show a peculiar response to eye opening background illumination can result in decreased alpha rhythm amplitude. ${ }^{7,8}$ Various investigations, using different combinations of electrodes in different montages and at various locations such as scalp surface, intracortical, intrathalamic, have been done to explore the properties of alpha rhythm. ${ }^{9}$ These experimental findings led to the conclusion that, in addition to the influence of some thalamic nuclei (mainly the pulvinar) on the generation of alpha rhythms over the occipital cortex, there are other intracortical network of neurons that take part in spread of alpha oscillations over the adjacent region. It seems that many small parts of the cortex (epicentres) give rise to multiple oscillations, which ultimately spread slowly at the rate of about $0.3 \mathrm{~cm} \mathrm{~s}^{-1}$. The cellular mechanisms responsible for the generation of alpha rhythms have recently been unveiled using in vitro preparations of thalamic nuclei. ${ }^{10}$ Hughes and Crunelli (2005) discovered that the occurrence of these oscillations depends on the activity of a subset of thalamocortical (TC) neurons termed high-threshold bursting cells, which are interconnected via gap junctions. These in vitro thalamic alpha rhythms can slow down until the theta frequency range when the TC neuron population is less depolarised. ${ }^{11}$ Besides being produced from occipital region, alpha frequency is generated in multiple cortical areas. Local cortical connections play important role in generating alpha rhythm, apart from the thalamocortical discharges which though have some influence on posterior dominant alpha rhythm. ${ }^{12}$ some recent work shows that pulvinar can coordinate communication between various brain areas and it is suggested that this area can have a pivotal role in prioritizing the processing of sensory information that is relevant for the task at hand. ${ }^{13}$

Theta Rhythm: The CA1 layer of the hippocampus gives rise to the strongest theta waves. The Entorhinal cortex via the entorhinal - CA1 pathway, is an important generator of this rhythm. Also, CA3-CA1, is another contributor to drive this rhythm, but it is actually not in phase with the first one i.e. input from entorhinal cortex. This leads to a gradual shift in phase with respect to depth within CA1. ${ }^{14}$ It has been observed that some short term memory tasks are associated with manifestation of theta rhythm. ${ }^{15}$ Also, recently Theta rhythm has been linked to the formation of long term memory in hippocampus. ${ }^{16}$ Some researches state that theta waves depicts the "on line" mode of the hippocampus, which means hippocampus can process any information coming at the moment. ${ }^{17}$ Experiments done on rodent hippocampi and entorhinal, show increased generation of theta waves during any memory recall and learning procedure, also these are important in inducing long term potentiation, a crucial step in the process of learning and memory. It has been shown in some of the electrophysiological studies that this theta rhythm has a potential role to keep the processes of memory recall and encoding of current sensory stimuli separate. Otherwise it would lead to lot of interruptions, if both of these processes (encoding sensory information and memory recall) run simultaneously. ${ }^{14,18}$

Apart from studies done on animals, few studies done on epileptic patients with electrodes being implanted intracranially, as a treatment modality, gives only little information about the human hippocampi theta waves. Cantero et al, found that hippocampus and neocortex do emit oscillations between 4-7 $\mathrm{Hz}$ frequency. ${ }^{19}$

Rapid eye movement and transition from sleep to awakening were related to rhythm from hippocampi. Also these were found in short bursts, duration being less than a second. No correlation could be found between cortical and hippocampal theta rhythms, it was proposed that probably these two mechanisms are controlled independently. Theta waves found in areas such as hippocampus, entorhinal cortex and cingular area (cortical limbic areas) are generally described here as rhythm slow activity (RSA). Studies suggest that septohippocampal cholinergic system (driven from brainstem reticular core) is the prime structure controlling at least one type of theta activity. ${ }^{20}$ local hippocampal circuits also actively contribute to the genesis of theta waves. This was shown by Konopacki et 
al., 1987 that application of cholinergic agonist in hippocampal slices produces waves within frequency range of theta rhythm..$^{21}$ one of the recent study has demonstrated that low frequency oscillations in the theta and alpha range provide a mechanism to functionally bind the hippocampus, prefrontal cortex and striatum during successful recollection of information. ${ }^{22}$

Delta Rhythm: This wave has high amplitude and its frequency is between $0.5-4$ hertz. These are recorded with electroencephalogram ${ }^{23}$ have been associated with deep stage 3 of NREM sleep. Source of delta wave has been attributed to either thalamus or cortex. Reticular formation also plays some role in coordinating the thalamus in generation of delta wave. ${ }^{24,25}$ It has also been shown in some of the work that damage to suprachiasmatic nuclei leads to disruption of delta wave activity. A right hemispherical dominance of delta waves has been observed during sleep. ${ }^{26}$ Also, role of T type calcium channels has been delineated in occurrence of delta waves. ${ }^{27}$ It has been observed that neurons are inhibited by the neurotransmitter gammaaminobutyric acid (GABA), during delta wave sleep, ${ }^{28}$ delta wave increases secretion of growth hormone releasing hormone GHRH and prolactin (PRL) and decreases the release of thyroid stimulating hormone (TSH). ${ }^{29}$ Recent work has shown that at low frequencies, simple phenomenon like eye closure causes widespread power increase involving all lobes of the brain and effect is evident in all frequency bands. On the contrary at high frequency, eye closure causes only focal power decrease over occipital cortex and frontal Brodmann area 8 and 9. ${ }^{30}$

\section{EEG During Sleep and Various Interventions}

Sleep EEG Phenomena: In the neurophysiology of sleep, two classic EEG phenomena have been established: the spindles or waves between 7 and $14 \mathrm{~Hz}$, which appear at sleep onset, and the delta waves (1-4 $\mathrm{Hz}$ ), which are paradigmatic of deeper stages of sleep. Experimental evidence has demonstrated that the sleep spindle oscillations are generated in the thalamus since they can be recorded in this brain area after decortication and high brain stem transection. Interestingly, we may note that the rhythmicity of the very slow oscillation appears to be reflected in that of the typical K-complexes of human EEG during nonREM sleep. ${ }^{31}$ During sleep thalamic pacemaker cells in the nucleus reticularis of thalamus stimulate thalamocortical cells which send excitatory impulses to the cortex. The spindle activity of these pacemaker cells within nucleus reticularis remains intact when they are surgically extracted whereas sleep spindle activity in the cortex ceases.

EEG during Activation Mechanisms: Various procedures are commonly used during EEG recording to increases the diagnostic yield of EEG. Common procedures are Hyperventilation (HV), Photic stimulation (PS), Sleep deprivation.
EEG during Hyperventilation (HV: Various studied have been conducted to observe the effect of $\mathrm{HV}$ on EEG. ${ }^{32}$

Hyperventilation mechanism was much utilized in the diagnosis of absence seizure. The response may depend upon age and effort put in by the patient. The common responses include no change or mild slowing in adults. There may be burst of delta waves exaggerated by prolonged hypoglycaemia. The proposed mechanism for hyperventilatory yield in EEG record is that there is rise in partial pressure of Oxygen and fall in partial pressure of carbon dioxide gas. Due to hypocapnia, there occurs constriction in the cerebral blood vessels leading to cerebral hypoperfusion, which increases the excitability of the neurons. Hypocapnia and respiratory alkalosis increase synchronous discharge from thalamocortical projections and concurrent decrease in activity of mesenchephalic reticular formation.

EEG during Photic Stimulation: Intermittent Photic stimulation or photic stimulation comprises of a series of short exposure to flashes of light. It requires that the patient should be attentive during the procedure. Normally, photic driving and no response is seen at all ages. Photic driving refers to a cerebral response which is time locked to the rate of light flashes. Mostly it is seen over the occipital region (O1 \& $\mathrm{O} 2$ electrode). It usually corresponds with the frequency which is close to patient's alpha rhythm.

Significant asymmetry in Photic driving is accepted when it occurs throughout the entire range of flashes, and present in resting EEG. ${ }^{33}$

Gender and EEG: Researchers have tried to study the effect of gender on various aspects of EEG. Nanbu Y et al studied Sex-related changes in the EEG in 15 male and 15 female healthy young adults, in two phases of rest and photic stimulation. ${ }^{34}$ They found inter hemispheric differences among male and female on EEG during photic stimulation. They stated that sex related differences occur with respect to lateralization of hemispheric function. Also, Richard J. Davidson et al studied EEG symmetry in males and female during self generated affective and cognitive tasks in three experiments. Their results showed that there is better control of asymmetrical cortical patterning in females as compared to males. It occurs that there is greater bilateral flexibility in females during self generation tasks. ${ }^{35}$

\section{Conclusion}

EEG is a useful, noninvasive, safe technique to study the electrical activity of brain in various physiological and pathological conditions. The rhythm of EEG can be correlated with the level of activation of the brain. Interventions like hyperventilation and photic stimulation can be used to increase the diagnostic yield of EEG. Finally, it has been seen that sex-related differences do exist in the degree of lateralization of hemispheric function 


\section{References}

1. Niedermeyer, E., Lopes da Silva, F. H., 1993. Electroencephalography: Basic principles, clinical applications and related fields, 3rd edition, Lippincott, Williams \& Wilkins, Philadelphia.

2. Caton R (1875) The electric currents of the brain. Br Med J. 2:278.

3. Berger H (1929) Über des Elekrenkephalogramm des Menschen. Arch Psychiat Nervenkr. 87(1):527-70.

4. Adrian ED, Mathews BHC (1934) The interpretation of potential waves in the cortex. J Physiol. 81(4):440-71.

5. Buser P, Rougeul-Buser A (2005) Visual attention in behaving cats: attention shifts and sustained attention episodes are accompanied by distinct electrocortical activities. Behav Brain Res. 164(1):42-51.

6. Rougeul A, Bouyer JJ, Dedet L, Debray O (1979) Fast somato-parietal rhythms during combined focal attention and immobility in baboon and squirrel monkey. Electroencephalogr Clin Neurophysiol. 46(3):310-9.

7. Cram JR, Kohlenberg RJ, Singer M (1977) Operant control of alpha EEG and the effects of illumination and eye closure. Psychosom Med. 39(1):11-8.

8. Paskewitz DA, Orne MT (1973) Visual effects on alpha feedback training. Science. 181(97):360-3.

9. Lopes da Silva F (1991) Neural mechanisms underlying brain waves: from neural membranes to networks. Electroencephalogr Clin Neurophysiol. 79(2):81-93.

10. Hughes SW, Lörincz M, Cope DW, Blethyn KL, Kékesi KA, Parri HR, Juhasz G, Crunelli V. Synchronized oscillations at alpha and theta frequencies in the lateral geniculate nucleus. Neuron. (2004)42(2):253-68.

11. Hughes SW, Crunelli V Thalamic mechanisms of EEG alpha rhythms and their pathological implications. Neuroscientist. (2005) 11(4):357-72.

12. Piotr Olejniczak. Neurophysiologic Basis of EEG. J Clin Neurophysiol. 2006;23(3):186-189.

13. Quax S, Jensen O, Tiesinga P. Top- down control of cortical gamma-band communication via pulvinar induced phase shifts in the alpha rhythm. PLoS Comput Biol 2017. 13(5):e1005519. https://doi.org/10.1371/journal.pcbi.1005519

14. Brankack, J; Stewart M, Fox SE. "Current source density analysis of the hippocampal theta rhythm: Associated sustained potentials and candidate synaptic generators". Brain Res. (1993)615(2):310327. doi:10.1016/0006-8993(93)90043M. PMID 8364740

15. Van Toller, S., Behan, J.M., Howells, P., Kendall-Reed, M. and Richardson, A. (1993) An analysis of spontaneous human cortical EEG activity to odours. Chem. Senses, 1993;18(2):1-16.

16. Berens, Sam C. et al._Theta Rhythm: Temporal Glue for Episodic Memory Current Biology, 2017:27(20) R1110R1112.

17. Buzsáki, G (2002). "Theta oscillations in the hippocampus". Neuron. 33(3):325-40

18. Hyman, JM; Wyble BP, Goyal V, Rossi CA, Hasselmo ME (December 17, 2003). "Stimulation in hippocampal region CA1 in behaving rats yields LTP when delivered to the peak of theta and LTD when delivered to the trough". J Neurosci. 23(37):11725-31. PMID 14684874.

19. Cantero JL, Atienza M, Stickgold R, Kahana MJ, Madsen JR, Kocsis B (2003). "Sleep-dependent theta oscillations in the human hippocampus and neocortex". $J$ Neurosci. 23 (34):10897-903.PMID 14645485

20. Petsche, H., Gogolak, G., and van Zwieten, P.A.. Rhythmicity of septal cell discharges at various levels of reticular excitation. Electroencephalogr. Clin
Neurophysiol. 1965. 19(1):25-33.

https://doi.org/10.1371/journal.pcbi.1005519

21. Konopacki, J., Bland, B.H., MacIvar, M., et al. Cholinergic theta rhythm in transected hippocampal slices: independent CA1 and dentate generators. Brain Res. 1987. 436(2):217-22.

22. Nora A. Herweg, Thore Apitz, XGregor Leicht, Christioph Mulert, Lluís Fuentemilla, and Nico Bunzeck. Theta-Alpha Oscillations Bind the Hippocampus, Prefrontal Cortex, and Striatum during Recollection: Evidence from Simultaneous EEG-fMRI. J Neurosci. 2016 Mar 23;36(12):3579-87.

23. Walker, Peter (1999). Chambers dictionary of science and technology. Edinburgh: Chambers. p. 312. ISBN 0-55014110-3.

24. Gross, Richard E. (1992). Psychology: the science of mind and behaviour. London: Hodder \& Stoughton. pp. 112-113. ISBN 0-340-56136-X.

25. Maquet, P., Degueldre, C., Delfiore, G., Aerts, J., Peters, J. M., Luxen, A., et al. (1997). Functional neuroanatomy of human slow wave sleep. Journal of Neuroscience. 17(8), 2807-2812.

26. Pilon M; Zadra A; Joncas S et al. Hypersynchronous delta waves and somnambulism: brain topography and effect of sleep deprivation. SLEEP. 2006;29(1):77-84.

27. Feinberg, I., T. Baker, R. Leder, and J. D. March. "Response of Delta (0-3 Hz) EEG and Eye Movement Density to a Night with 100 Minutes of Sleep." Sleep. (1988);11(5):473-87.

28. Kryzhanovskii, G. N., A. A. Shandra, L. S. Godlevskii, and I. I. Mikhaleva. "Appearance of Parkinsonian Syndrome after Administration of Delta Sleep-inducing Peptide into the Rat Substantia Nigra." Biull Eksp Biol Med. (1990);109(2):119-21.

29. Alfimova, M. V., \& Uvarova, L. G. (2007). Changes in the EEG spectral power during perception of neutral and emotionally salient words in schizophrenic patients, their relatives and healthy individuals from general population. Zh Vyssh Nerv Deiat Im I P Pavlova. 2007 JulAug;57(4):426-36.

30. Aaron S. Geller, John F. Burke, Michael R. Sperling, Ashwini D. Sharan, Brian Litt, Gordon H. Baltuch, et al. Eye closure causes widespread low-frequency power increase and focal gamma attenuation in the human electrocorticogram. Clin Neurophysiol. 2014;125(9):1764-1773.

31. Amzica F, Steriade M (1997) The K-complex: its slow $(<1-\mathrm{Hz})$ rhythmicity and relation to delta waves. Neurology, 49(4):952-9.

32. Rockstroh B; Hyperventilation-induced EEG changes in humans and their modulation by an anticonvulsant drug: Epilepsy Res. 1990 Nov;7(2):146-54.

33. Sullivan, L.; Activation Procedures, Part II Photic Stimulation: ASET news Volume 29, Number 4.

34. Nanbu Y, Wada Y, Kadoshima R, Kitazawa S, Futamata $\mathrm{H}$, Hashimoto T, Koshino Y; Sex-related differences in EEG coherence in normal young adults--evaluation during rest and photic stimulation: Rinsho Byori. 1995 Jul;43(7):713-7.

35. Davidson, Richard J. et al. Sex difference in patterns of asymmetry. Biological Psychology. (1976);4(2)119-138.

\begin{tabular}{|l|}
\hline How to cite this article: Ojha P, Pandey N, Singh \\
S. Cortical generators of normal \\
electroencephalographic waveforms: A literature \\
review. Ind J Clin Anat Physiol. 2018;5(3):291-294. \\
\hline
\end{tabular}

